\title{
Identification of circulating hub long noncoding RNAs associated with hypertrophic cardiomyopathy using weighted correlation network analysis
}

\author{
QI GUO, JUNJIE WANG, RUNLU SUN, WENLI GU, ZHIJIAN HE, QIAN CHEN, \\ WENHAO LIU, YANGXIN CHEN, JINGFENG WANG and YULING ZHANG \\ Department of Cardiology, Sun Yat-sen Memorial Hospital of Sun Yat-sen University, \\ Guangzhou, Guangdong 510000, P.R. China
}

Received April 15, 2020; Accepted September 7, 2020

DOI: $10.3892 / \mathrm{mmr} .2020 .11566$

\begin{abstract}
Hypertrophic cardiomyopathy (HCM) is one of the most commonly inherited heart diseases and the leading cause of sudden cardiac death among adolescents and young adults. Circulating long noncoding RNAs (lncRNAs) have demonstrated potential as diagnostic and therapeutic targets in several cardiovascular diseases. However, the circulating extracellular lncRNA expression profile of patients with HCM remains unclear. Plasma lncRNA expression was evaluated in patients with HCM and healthy controls using a human lncRNA microarray. A weighted correlation network analysis (WGCNA) and linear models for microarray data (Limma) were used. GSE68316 data from cardiac tissue in the Gene Expression Omnibus database were analysed for further validation. Using WGCNA, two modules (referred to as the magenta and the light-yellow module) were identified that were positively associated with HCM. Gene Ontology analysis revealed that lncRNAs in the magenta module targeted 'heart growth'. Using Limma, a total of 290 lncRNAs were differentially expressed (210 upregulated and 80 downregulated) in the plasma of HCM patients, compared with controls. Moreover, combined WGCNA and Limma analysis demonstrated that 27 hub lncRNAs in the magenta module and 13 hub lncRNAs in the light-yellow module were significantly upregulated, compared with the controls. Moreover, of the 40 differentially expressed hub IncRNAs identified in the two modules, three circulating lncRNAs (lnc-P2RY6-1:1, ENST00000488040 and ENST00000588047) were also significantly upregulated
\end{abstract}

Correspondence to: Dr Yuling Zhang or Dr Jingfeng Wang, Department of Cardiology, Sun Yat-sen Memorial Hospital of Sun Yat-sen University, 127 Yanjiangxi Road, Guangzhou, Guangdong 510000, P.R. China

E-mail: zzhangyuling@126.com

E-mail: drwjf@hotmail.com

Key words: long noncoding RNA, weighted correlation network analysis, hypertrophic cardiomyopathy in the HCM cardiac tissue validation dataset. These lncRNAs may serve as biomarkers and therapeutic targets for precise diagnosis and treatment of HCM.

\section{Introduction}

Hypertrophic cardiomyopathy (HCM) is one of the most commonly inherited heart diseases, with a prevalence of one in 167 people (1). It is the leading cause of sudden cardiac death among adolescents and young adults, especially athletes (2). HCM is an autosomal dominant genetic disorder caused predominantly by mutations in sarcomere genes, such as myosin binding protein $\mathrm{C} 3(M Y B P C 3)$ and myosin heavy chain 7 (MYH7) (3). However, disease-causing sarcomere mutations are absent in $\sim 70 \%$ of patients with established disease, and sarcomere gene carriers can live to advanced ages without developing disease (4). Thus, expanding the focus of HCM research beyond DNA mutations towards epigenetic determinants, such as noncoding RNA, may provide insight into the pathogenesis of this disease (4).

Long noncoding RNAs (lncRNAs) constitute a heterogeneous class of transcripts $>200$ nucleotides in length (5). Compared with coding mRNA, most lncRNAs cannot code proteins and have previously been regarded as genomic transcriptional noise. Nevertheless, increasing evidence suggests that lncRNAs are involved in several biological processes and diseases, such as heart failure, acute myocardial fibrosis and atherosclerosis (6). IncRNAs have been implicated in the pathological processes of HCM, including myocardial hypertrophy, cardiomyocyte disarrangement and interstitial fibrosis (7). A previous microarray study identified several dysregulated lncRNAs in myocardial tissue from patients with HCM, compared with control subjects (8). However, changes in circulating extracellular lncRNAs, which are an emerging class of biomarkers relevant to myocardial biology (9), have not been determined in HCM.

Weighted gene co-expression network analysis (WGCNA) is a novel and powerful systems biology method that is increasingly used in bioinformatics analysis of microarray profiling data (10). Using this method, highly correlated genes are clustered into modules, and several modules form a gene 
co-expression network. After relating modules to clinical traits, biologically relevant modules can be identified in any given disease. Compared with standard comparative analysis, WGCNA can identify critical genes with key roles in the phenotype and development of a disease from interesting modules associated with important clinical traits (11). Despite the strengths and popularity of network analysis, WGCNA has not been employed to analyse lncRNA microarray data in HCM. Therefore, in the present study, WGCNA and further analyses were performed to identify circulating hub lncRNAs associated with HCM. The aim of the study was to identify potential biomarkers and therapeutic targets for improved diagnosis and treatment of HCM.

\section{Materials and methods}

Study population. The study population consisted of $14 \mathrm{HCM}$ patients and 7 healthy control subjects at Sun Yat-sen Memorial Hospital of Sun Yat-sen University from October 2018 to May 2019. HCM diagnosis was carried out according to the European Society of Cardiology Guidelines (12). HCM was defined as wall thickness $\geq 15 \mathrm{~mm}$ in one or more left ventricular myocardial segments in individuals with no history of HCM, or a wall thickness $\geq 13 \mathrm{~mm}$ in one or more left ventricular myocardial segments in individuals with an HCM history in a first-degree relative, as measured by echocardiography or cardiac magnetic resonance (12). Patients with a history of ventricular septal surgery, valvular heart disease, coronary artery disease, atrial fibrillation, systemic hypertension, diabetes, surgery or trauma within six months, cancer or renal dysfunction were excluded from the study.

This study was carried out in accordance with the recommendations of the ethics committee of Sun Yat-sen Memorial Hospital, and all subjects provided written informed consent in accordance with the Declaration of Helsinki.

Plasma collection and microarray. Whole blood was collected in venous blood collection tubes containing EDTA after overnight fasting, and plasma was separated by centrifugation. Total RNA (250 ng) was isolated using a Plasma RNA Purification Mini kit (Norgen Biotek Corp.) according to the manufacturer's instructions and purified using an RNeasy Mini kit (Qiagen $\mathrm{GmbH}$ ). RNA integrity was examined using an Agilent Bioanalyzer 2100 (Agilent Technologies, Inc.). RNA samples from each group were then used to generate biotinylated cRNA targets for the LC Human ceRNA array (version 1.0; LC-Bio Technology Co., Ltd.). In brief, RNA was reverse transcribed into first strand of cDNA with Promoter Primer by AffinityScript-RT Kit (Agilent Technologies, Inc.). The second strand of cDNA was generated with Anti-sense Promotor. This cDNA was used as a template to synthesize cRNA by the T7 RNA polymerase mix. The biotinylated cRNA targets were then hybridized with slides for $17 \mathrm{~h}$ at $65^{\circ} \mathrm{C}$. After hybridization, the slides were scanned on an Agilent Microarray Scanner (Agilent Technologies, Inc.). Data were extracted using Feature Extraction software (version 12.0.3.1; Agilent Technologies, Inc.). Raw data were normalized with the quantile algorithm using Genespring software (version 14.8, Agilent Technologies, Inc.). The microarray experiments were performed following the protocol of Agilent Technologies Inc. by LC Sciences Corporation. All microarray data have been uploaded in the Gene Expression Omnibus (GEO) database under the accession number GSE143786 (https:/www.ncbi. nlm.nih.gov/geo/query/acc.cgi?acc=GSE143786). After microarray data collection, bioinformatic analysis and validation were performed according to the flow chart presented in Fig. 1.

WGCNA. The WGCNA R package (version 1.68, https:// cran.r-project.org/web/packages/WGCNA/index.html) was used to construct a weighted correlation network between lncRNAs, as described previously (10). In the network, pairwise Pearson coefficients were calculated to evaluate the weighted co-expression relationship between all genes in the adjacency matrix. To ensure a scale-free network, a soft threshold of $\beta$ was used to guarantee the minimum scale free topology fitting index $\mathrm{R}^{2}$ as 0.8 . The topological overlap measure (TOM) was used to represent the network interconnectedness. Gene modules consisting of genes with high correlation were detected using the hierarchical clustering method, based on a TOM-based dissimilarity measure (1-TOM) (13). Each module was assigned a unique colour. Hierarchical clustering dendrograms were identified using the dynamic branch cut method in WGCNA (14).

Identification of clinically relevant modules and functional annotation. Gene significance (GS), module significance (MS) and module eigengene (ME) were calculated. Briefly, GS was defined as-log (P-value) of a gene, MS was the average GS across the module, and the ME was the first principal component of a given module. The significance between the ME and interested clinical trait was also calculated, as modules with high trait significance $(\mathrm{P}<0.05)$ may be highly associated with disease (15). Modules from hierarchical clustering with significant correlations with clinical traits were selected as key modules, under the criteria of correlation efficient $\geq 0.55$ and $\mathrm{P}<0.05$, for subsequent analysis. For each key module, intramodular analysis was carried out to evaluate the association between module membership (correlation efficient between lncRNA expression and ME) and GS for HCM, thickness, obstruction, and left atrial diameter using Pearson correlation method. Gene ontology (GO) functional annotation using the ClueGO app in Cytoscape (version 3.7.2; https:// cytoscape.org/) was performed to identify the potential functions and underlying mechanisms associated with selected modules $(16,17)$.

Identification and validation of differentially expressed hub lncRNAs. Hub genes in key modules were defined as genes with an absolute value of the module membership $>0.8$, the correlation efficient between lncRNA and clinical trait $>0.2$ and the weighted P-value of association with the trait $<0.01$ (18). Differentially expressed lncRNAs were identified using the Linear Models for Microarray data (Limma) package (version 3.34.9) in R (19). Volcano plot and heatmap were generated using $\mathrm{R}$ tool to shown differential expressed IncRNAs explicitly. The z-score for each lncRNA was calculated by subtracting the mean level from the raw expression level, then dividing the difference by the standard deviation. Differentially expressed hub lncRNAs were obtained by mapping hub lncRNAs against differentially expressed 




Figure 1. Study design flow chart. HCM, hypertrophic cardiomyopathy; lncRNA, long noncoding RNA; WGCNA, weighted gene co-expression network analysis; Limma, Linear Models for Microarray data; lncRNA, long noncoding RNA.

IncRNAs. The GSE68316 lncRNA microarray was obtained from the GEO database using cardiac samples and used for validation of these differentially expressed hub lncRNAs using the Limma package in R. Each differentially expressed hub IncRNA in our own dataset was evaluated whether it was also significantly dysregulated in GSE68316 dataset. Benjamini-Hochberg adjustment for P-values was applied to the Limma analysis.

Statistical analysis. Categorical variables are presented as $\mathrm{n}(\%)$, and continuous variables as the mean \pm SD. Student's t-test and Fisher's exact probability test were used to compare differences between the HCM group and the control group. All statistical analyses were performed with the $\mathrm{R}$ tool (version 3.6.1; https://www.r-project.org/). P<0.05 was considered to indicate a statistically significant difference.

\section{Results}

Baseline characteristics of the study participants. A total of 14 patients with HCM patients and seven control subjects were enrolled in the present study. The average age of all enrolled subjects was $58.85 \pm 2.12$ years (data not shown). No significant differences in age, sex, or body mass index were observed between the two groups. Total cholesterol levels were significantly increased $(\mathrm{P}<0.001)$, and blood glucose levels significantly reduced $(\mathrm{P}=0.028)$ in the HCM group, compared with the control group. Cardiac ultrasound examination indicated that the maximum left ventricular wall thickness was significantly increased in patients with HCM, compared with healthy controls $(20.00 \pm 4.52$ and $9.28 \pm 1.25 \mathrm{~mm}$, respectively; $\mathrm{P}<0.001$; Table SI).
Data pre-processing and co-expression network construction. To construct a co-expression network, all 16,996 lncRNAs obtained with 21 plasma samples by microarray were used in the following analysis. In the present study, the soft-thresholding power was determined to be $\beta=7$, where the scale-free topology fit curve first reached $\mathrm{R}^{2}=0.8$, to construct a weighted network based on a scale-free topology criterion, aiming to help detect modules in following hierarchical clustering (Fig. S1). A total of 27 modules were detected through the dynamic tree cutting method (Fig. S2). The turquoise module was the largest module, consisting of 5,630 lncRNAs, while the dark orange module was the smallest module, with only 35 lncRNAs.

Identification of clinically relevant modules associated with $H C M$. After mapping modules to clinical traits, correlations and significance were observed for the HCM trait, maximum left ventricular wall thickness, left ventricular outflow tract obstruction, and left atrial diameter (Fig. 2). The highest correlations with the HCM trait were observed in the magenta $(\mathrm{r}=0.66 ; \mathrm{P}=0.001)$ and $\tan$ module $(\mathrm{r}=-0.66 ; \mathrm{P}=0.001)$. Maximum left ventricular wall thickness was significantly correlated with the light-yellow ( $\mathrm{r}=0.55 ; \mathrm{P}=0.009)$, magenta $(\mathrm{r}=0.55 ; \mathrm{P}=0.01)$ and tan modules $(\mathrm{r}=-0.57 ; \mathrm{P}=0.007)$. The magenta $(r=0.59 ; \mathrm{P}=0.005)$ and brown modules $(\mathrm{r}=0.63$; $\mathrm{P}=0.002)$ were significantly correlated with left ventricular outflow tract obstruction.

Similarly, in eigengene adjacency dendrograms, the magenta, light-yellow and salmon modules were associated with HCM, maximum left ventricular wall thickness, and left ventricular outflow tract obstruction. Heatmap plot of the adjacencies in the eigengene network showed a high adjacency between HCM and magenta module (Fig. 3).

Given the evidence from both module-trait correlations and eigengene adjacency dendrograms, the magenta and the light-yellow modules were selected for subsequent analysis.

Intramodular analysis and functional annotation of modules. For 446 lncRNAs in the magenta module, module membership positively corelated with GS for HCM ( $\mathrm{r}=0.33$; $\left.\mathrm{P}=8.1 \times 10^{-13}\right)$, thickness $\left(\mathrm{r}=0.36 ; \mathrm{P}=4.0 \times 10^{-15}\right)$ and obstruction $\left(\mathrm{r}=0.51 ; \mathrm{P}=5.7 \times 10^{-31}\right)$. A negative correlation was observed between module membership and GS for left atrial diameter $\left(\mathrm{r}=-0.27 ; \mathrm{P}=6.6 \times 10^{-9}\right)$, according to intramodular analysis (Fig. S3).

Similarly, for 65 lncRNAs in the light-yellow module, positive correlations were observed between module membership and gene significance for $\mathrm{HCM}\left(\mathrm{r}=0.42, \mathrm{P}=5 \times 10^{-4}\right)$ and thickness $(\mathrm{r}=0.31 ; \mathrm{P}=0.012$; Fig. $\mathrm{S} 4)$. Functional annotation demonstrated that the magenta module was enriched in $\mathrm{GO}$ terms, such as 'heart growth', 'cardiac muscle tissue growth' and 'regulation of coagulation' (adjusted $\mathrm{P}<0.05$; Fig. S5).

Identification and validation of differentially expressed hub lncRNAs. Based on the screening criteria for hub lncRNA, 74 hub lncRNAs and 42 hub lncRNAs were selected from the magenta module and light-yellow module, respectively (Tables SII and SIII). A correlation heatmap for these 116 hub lncRNAs indicated general positive correlations between each two hub lncRNAs (Fig. 4). 


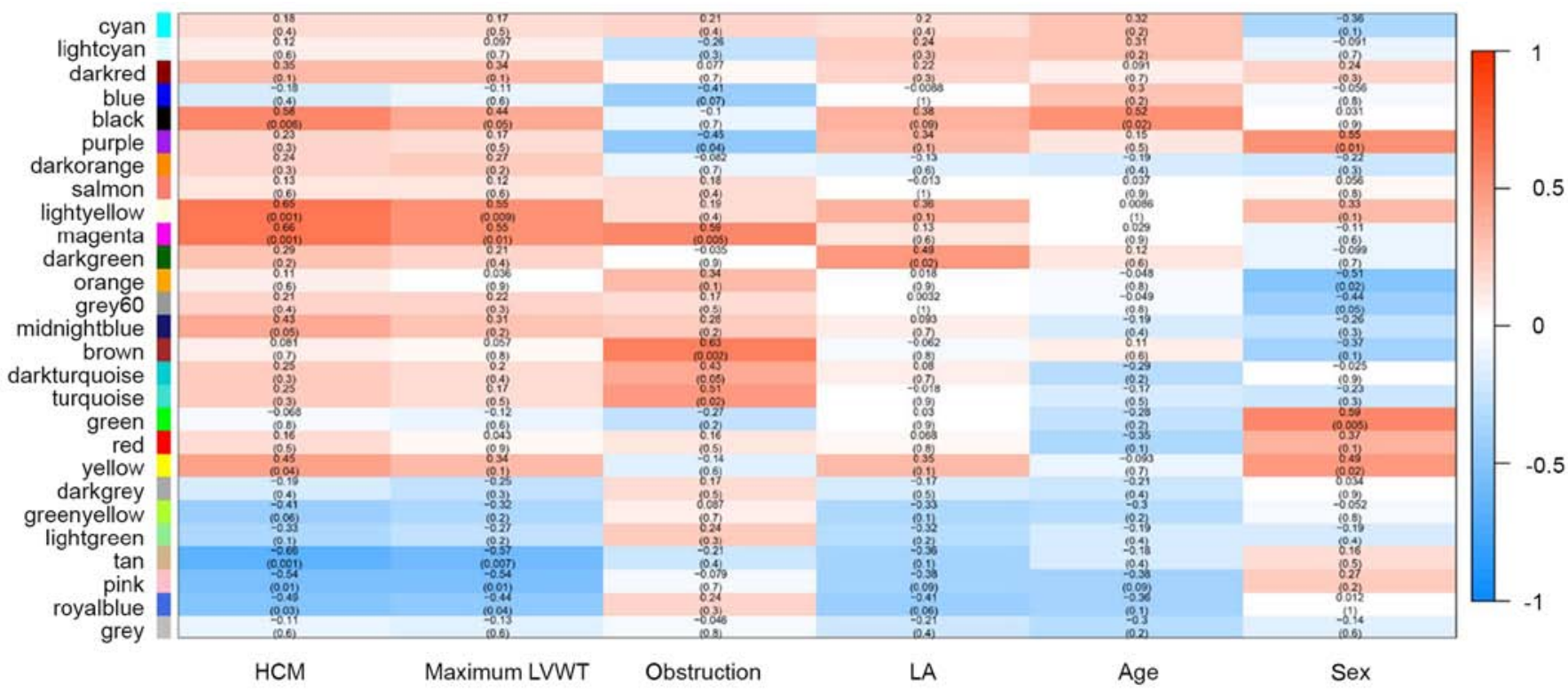

Figure 2. Module-trait associations. Each row corresponds to a module eigengene, and each column a clinical trait. The corresponding correlation coefficient (top) and P-value (bottom) for each module-trait pair is displayed in each cell. The correlation coefficient was assigned a color according to a color scale ranging from-1 (blue) to 1 (red). HCM, hypertrophic cardiomyopathy; LVWT, left ventricular wall thickness; LA, left atrial diameter.


Figure 3. Eigengene dendrogram and heatmap identifying groups of correlated eigengenes. The dendrogram indicates that the magenta and light-yellow modules are highly related to HCM and maximum left ventricular wall thickness. Eigengene adjacency was assigned a color according to a color scale ranging from 0 (blue) to 1 (red). HCM, hypertrophic cardiomyopathy; LA, left atrial diameter.

A total of 80 downregulated lncRNAs and 210 upregulated lncRNAs were identified using Limma (Fig. 5A). The HCM and control groups were distinguishable by hierarchical clustering of differentially expressed lncRNAs (Fig. 5B). The hub genes from each module were mapped with all differentially expressed IncRNAs identified between the HCM and the 


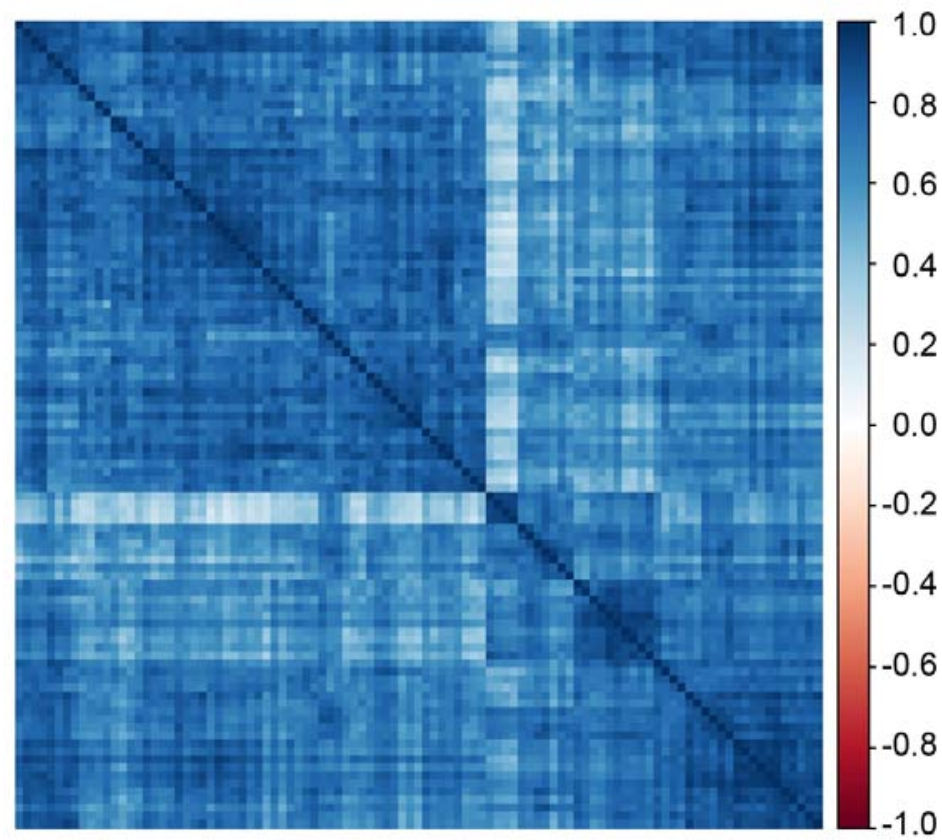

Figure 4. Hub lncRNA correlation plot. The correlation plot illustrates the correlation matrix among hub lncRNAs in the co-expression network analysis. Each row or column corresponds to one lncRNA. The correlation coefficient for lncRNA pairs was assigned a color according to a color scale ranging from-1 (blue) to 1 (red). LncRNA, long non-coding RNA.
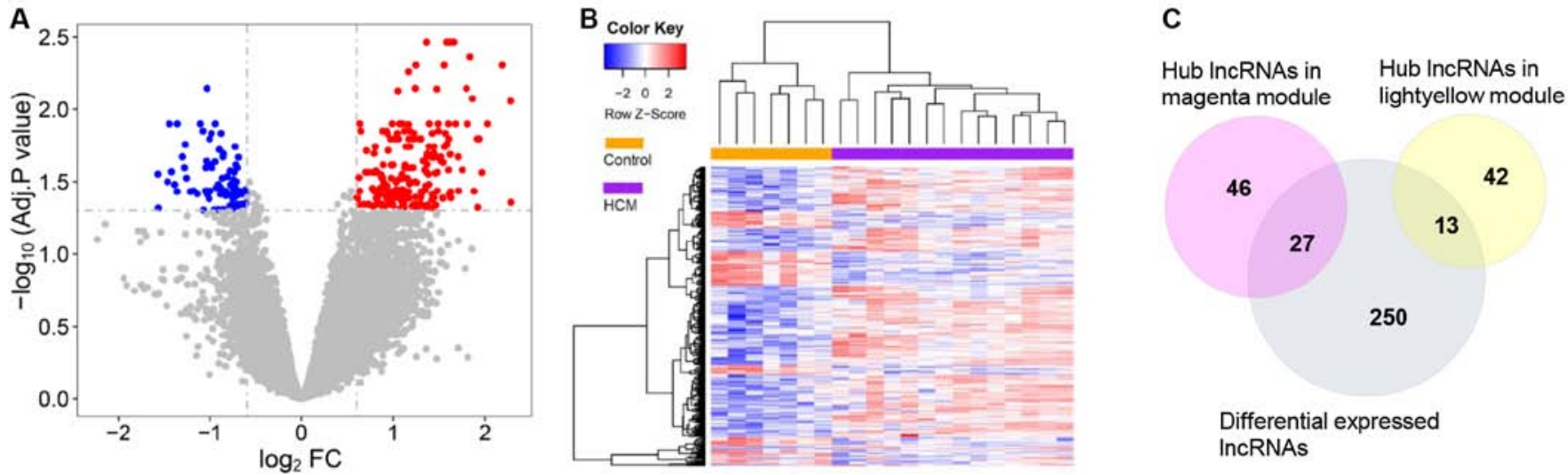

Figure 5. Identification and validation of differentially expressed hub lncRNAs associated with HCM. (A) Volcano plot. A total of 80 downregulated and 210 upregulated lncRNAs were identified using the Limma package. (B) Heatmap. Differentially expressed lncRNAs were identified in the HCM groups relative to the controls. Each lncRNA was assigned a color according to Z-score scale. (C) Venn diagram. Differentially expressed hub lncRNAs were obtained by mapping hub lncRNAs against differentially expressed lncRNAs. HCM, hypertrophic cardiomyopathy; FC, fold-change; adj.P, P-value after Benjamini-Hochberg adjustment; lncRNA, long noncoding RNA.

control groups. As a result, 27 hub lncRNAs in the magenta module and 13 hub lncRNAs in the light-yellow module were identified as differentially expressed (adjusted $\mathrm{P}<0.05$ by Limma; Table I).

The GSE68316 microarray dataset resulting from cardiac tissue from patients with HCM $(n=7)$ and controls $(n=5)$ was used for further validation. Among the 40 differentially expressed hub lncRNAs identified in the magenta and the light-yellow modules, four lncRNAs were also expressed in the cardiac tissue dataset in the HCM and control groups. Of these, lnc-P2RY6-1:1 ENST00000488040 and ENST00000588047 were significantly upregulated in the HCM group ( $\log _{2}$ fold change, 0.67, 0.76 and 0.60 , respectively; adjusted $\mathrm{P}<0.05)$, compared with the control group (Table II).
Functional annotation of 3 hub differentially expressed lncRNAs. To investigate the potential functions associated with the differentially expressed hub lncRNAs, the functional annotation was performed for P2RY6-1:1 ENST00000488040 and ENST00000588047. 'Cardiac ventricle morphogenesis' was associated with lnc-P2RY6-1:1. Moreover, ENST00000488040 was associated with 'positive regulation of cardiac muscle hypertrophy' and 'physiological cardiac muscle hypertrophy'. In addition, lnc-P2RY6-1:1 and ENST00000588047 were associated with immune responses. For instance, lnc-P2RY6-1:1 was associated with ' $\mathrm{T}$ cell differentiation involved in immune response', while ENST00000588047 was associated with 'B cell apoptotic process' and 'positive regulation of T cell migration' (Table SIV). 
Table I. Number of lncRNAs in the magenta and light yellow module.

\begin{tabular}{lcc}
\hline Number & Magenta module & Light yellow module \\
\hline lncRNAs & 446 & 65 \\
Hub lncRNAs & 73 & 42 \\
Differential expressed hub lncRNAs & 27 & 13 \\
Validated in GSE68316 & 3 & 1 \\
\hline
\end{tabular}

lncRNA, long noncoding RNA.

Table II. Four lncRNAs were validated in GSE68316 dataset by using cardiac tissue.

\begin{tabular}{lllcccc}
\hline IncRNAs & Module & Gene symbol & $\log _{2}(\mathrm{FC})$ & $\begin{array}{c}\text { Adjusted } \\
\text { P-value }\end{array}$ & $\begin{array}{c}\log _{2}(\mathrm{FC}) \text { in } \\
\text { GSE68316 }\end{array}$ & $\begin{array}{c}\text { Adjusted P-value } \\
\text { in GSE68316 }\end{array}$ \\
\hline Inc-P2RY6-1:1 & Light yellow & Inc-P2RY6-1 & 0.80 & $1.81 \times 10^{-2}$ & 0.67 & $3.30 \times 10^{-3}$ \\
ENST00000488040 & Magenta & RP11-550I24.2 & 1.18 & $1.41 \times 10^{-2}$ & 0.76 & $1.75 \times 10^{-4}$ \\
ENST00000588047 & Magenta & CTD-2006C1.2 & 0.87 & $2.81 \times 10^{-2}$ & 0.60 & $9.59 \times 10^{-4}$ \\
ENST00000587071 & Magenta & TP73-AS1 & 0.95 & $3.67 \times 10^{-2}$ & -0.10 & $4.70 \times 10^{-1}$ \\
\hline
\end{tabular}

lncRNA, long noncoding RNA; FC, fold-change.

\section{Discussion}

In the present study, a comprehensive analysis of lncRNAs was carried out using plasma transcriptome profiles from patients with HCM and healthy controls. By constructing a WGCNA network and calculating the co-expression relationships between IncRNAs, hub genes in key modules were identified, providing an overview of the expression profiles and functional relationships of these hub genes. To the best of our knowledge, the present study is the first to use the WGCNA to screen key lncRNA modules in HCM plasma. Two modules, the magenta and light-yellow modules, were strongly associated with HCM. In addition, functional annotation of the magenta module indicated an association with GO terms, such as 'cardiac tissue growth', suggesting a potentially important role in HCM. Moreover, hub genes within each module were identified using module membership, gene significance and weighted $\mathrm{q}$ thresholds. Among these hub genes, several differentially expressed hub lncRNAs in the trait-associated modules were identified, then validated in an independent dataset from cardiac tissue (8). A total of three validated hub lncRNAs (lnc-P2RY6-1:1, ENST00000488040, and ENST00000588047) from the two key modules were significantly differentially upregulated. Thus, the present study provided a comprehensive evaluation of lncRNA regulation in HCM and identified lncRNAs that may serve as biomarkers and therapeutic targets for HCM diagnosis and treatment.

As a primary cardiovascular inherited disease, HCM is the most common risk factor of sudden cardiac death among young people (2). Several mutations in gene-encoding regions are associated with HCM pathogenesis, with $M Y B P C 3$ and $M Y H 7$ sarcomere mutations potentially underlying approximately one-half of HCM cases (20). However, this observation also implies that disease-causing sarcomere mutations are absent in $\sim$ one-half of patients with established disease. Additionally, sarcomere gene mutation carriers can live to advanced ages without developing HCM, challenging the conventional single-gene hypothesis for HCM (4). Therefore, expanding the HCM research focus from causal DNA mutations towards additional epigenetic determinants of the HCM phenotype may provide new insight into the pathogenesis of this disease.

Several studies have demonstrated the important role of lncRNA in the different pathophysiological changes observed in HCM. For instance, the myosin heavy chain-associated RNA transcript (Mhrt) lncRNA was found to be cardiac-specific and abundant in adult hearts (21). Moreover, lncRNA Mhrt served a protective role in the heart against hypertrophy and cardiac failure by interacting with chromatin (21). Genetic variation at the IncRNA H19 gene is also associated with the predisposition to HCM (22). A small-sample lncRNA and mRNA microarray analysis of myocardial tissue from seven patients with HCM and five controls and identified 965 upregulated and 461 downregulated lncRNAs by comparative analysis (8). Functional enrichment analysis indicated that these dysregulated lncRNAs were associated with ribosome and oxidative phosphorylation (8).

Data mining can extract crucial biological molecule information from large datasets and identify molecules that may be used as potential biomarkers or targets to improve the diagnosis, treatment and prevention of certain diseases. As the most widespread method in gene expression analysis, traditional comparative differential expression analysis has a number of limitations. This method treats each gene separately and enrols only the most significant dysregulated genes 
while ignoring others, assuming that each gene is independent of all other genes (23). By contrast, network topology often considers global genes and can reveal more gene interaction information. Among network analysis methods, WGCNA is a systems biology method for describing correlation patterns among genes across microarray samples (10). These methods have been widely applied in various biological conditions, such as periodontitis (24), osteoarthritis (25) and liver regeneration (26). In the present study, WGCNA and the Limma method were used together to identify differentially expressed hub lncRNAs in HCM. Notably, previous studies enrolled parts of microarray data into WGCNA (25); however, this study entered all microarray data into the WGCNA rather than the differentially expressed data in previous studies, which may result in a more comprehensive correlation network according to the tutorial (10).

The present study has several limitations. First, the sample size was relatively small. Although a total of $14 \mathrm{HCM}$ patients and seven healthy control subjects were enrolled, representing a larger sample size than that in a previous study (8), a larger study cohort would provide further validation of the present findings. Moreover, type-I error resulting from a large number of analyses of the microarray data may exist. However, Benjamini-Hochberg adjustment for P-values in the Limma analysis was applied to reduced type-I errors. Lastly, as this study evaluated IncRNA expression profiles based solely on bioinformatics, functional experiments in cardiac tissue or cells, such as quantitative real-time PCR or western blotting, should be performed for further validation. Nevertheless, under the hypothesis that key plasma lncRNAs may be distinctly expressed in cardiac tissue from patients with HCM, cardiac tissue microarray data were used for validation, which already partly confirmed the present findings.

In conclusion, three differentially expressed hub lncRNAs (lnc-P2RY6-1:1, ENST00000488040, and ENST00000588047) in two key modules were identified in HCM that may serve as biomarkers and therapeutic targets for the precise diagnosis and treatment of $\mathrm{HCM}$ in the future.

\section{Acknowledgements}

Not applicable.

\section{Funding}

The present study was supported by grants from the National Natural Science Foundation for Young Scientists of China (grant no. 81700397) and the Guangzhou Science and Technology Plan Project (grant nos. 201704020044 and 201803040010).

\section{Availability of data and materials}

The datasets used and/or analysed during the current study are available from the corresponding author upon reasonable request, or in the Gene Expression Omnibus database under the accession numbers GSE143786 (plasma, https:/www. ncbi.nlm.nih.gov/geo/query/acc.cgi?acc=GSE143786) and GSE68316 (cardiac tissue, https://www.ncbi.nlm.nih.gov/geo/ query/acc.cgi?acc=GSE68316).

\section{Authors' contributions}

QG, JiW and YZ conceived the present study. JuW and WG collected and analysed the data. QG, RS and YC performed the experiments. ZH, WL and QC were responsible for analysis and visualization of the data. QG and WL wrote the original draft of the manuscript. YC, JiW and YZ reviewed and edited the manuscript. All authors have read and approved the manuscript.

\section{Ethics approval and consent to participate}

This study was carried out in accordance with the recommendations of the ethics committee of Sun Yat-sen Memorial Hospital (approval no 2018-KY-075). All subjects provided written informed consent in accordance with the Declaration of Helsinki.

\section{Patient consent for publication}

Not applicable.

\section{Competing interests}

The authors declare that they have no competing interests.

\section{References}

1. Semsarian C, Ingles J, Maron MS and Maron BJ: New perspectives on the prevalence of hypertrophic cardiomyopathy. J Am Coll Cardiol 65: 1249-1254, 2015.

2. Marian AJ and Braunwald E: Hypertrophic cardiomyopathy: Genetics, pathogenesis, clinical Manifestations, diagnosis, and therapy. Circ Res 121: 749-770, 2017.

3. Geske JB, Ommen SR and Gersh BJ: Hypertrophic cardiomyopathy: Clinical update. JACC Heart Fail 6: 364-375, 2018.

4. Maron BJ, Maron MS, Maron BA and Loscalzo J: Moving beyond the sarcomere to explain heterogeneity in hypertrophic cardiomyopathy: JACC review topic of the week. J Am Coll Cardiol 73: 1978-1986, 2019.

5. Sallam T, Sandhu J and Tontonoz P: Long noncoding RNA discovery in cardiovascular disease: Decoding form to function. Circ Res 122: 155-166, 2018.

6. Lucas T, Bonauer A and Dimmeler S: RNA therapeutics in cardiovascular disease. Circ Res 123: 205-220, 2018.

7. Sun $J$ and Wang C: Long non-coding RNAs in cardiac hypertrophy. Heart Fail Rev: Oct 29, 2019 (Epub ahead of print).

8. Yang W, Li Y, He F and Wu H: Microarray profiling of long Non-coding RNA (lncRNA) associated with hypertrophic cardiomyopathy. BMC Cardiovasc Disord 15: 62, 2015.

9. Shah RV, Rong J, Larson MG, Yeri A, Ziegler O, Tanriverdi K, Murthy V, Liu X, Xiao C, Pico AR, et al: Associations of circulating extracellular RNAs with myocardial remodeling and heart failure. JAMA Cardiol 3: 871-876, 2018.

10. Langfelder P and Horvath S: WGCNA: An R package for weighted correlation network analysis. BMC Bioinformatics 9: $559,2008$.

11. Zhang J, Nie Q, Si C, Wang C, Chen Y, Sun W, Pan L, Guo J, Kong J, Cui Y, et al: Weighted gene co-expression network analysis for RNA-sequencing data of the varicose veins transcriptome. Front Physiol 10: 278, 2019.

12. Elliott PM, Anastasakis A, Borger MA, Borggrefe M, Cecchi F, Charron P, Hagege AA, Lafont A, Limongelli G, Mahrholdt $\mathrm{H}$, et al: 2014 ESC guidelines on diagnosis and management of hypertrophic cardiomyopathy: The task force for the diagnosis and management of hypertrophic cardiomyopathy of the european society of cardiology (ESC). Eur Heart J 35: 2733-2779, 2014

13. Ravasz E, Somera AL, Mongru DA, Oltvai ZN and Barabási AL: Hierarchical organization of modularity in metabolic networks. Science 297: 1551-1555, 2002. 
14. Langfelder P, Zhang B and Horvath S: Defining clusters from a hierarchical cluster tree: The dynamic tree cut package for $\mathrm{R}$. Bioinformatics 24: 719-720, 2008.

15. Fuller TF, Ghazalpour A, Aten JE, Drake TA, Lusis AJ and Horvath S: Weighted gene coexpression network analysis strategies applied to mouse weight. Mamm Genome 18: 463-472, 2007.

16. Bindea G, Mlecnik B, Hackl H, Charoentong P, Tosolini M, Kirilovsky A, Fridman WH, Pages F, Trajanoski Z and Galon J: ClueGO: A Cytoscape plug-in to decipher functionally grouped gene ontology and pathway annotation networks. Bioinformatics 25: 1091-1093, 2009.

17. Shannon P, Markiel A, Ozier O, Baliga NS, Wang JT, Ramage D, Amin N, Schwikowski B and Ideker T: Cytoscape: A software environment for integrated models of biomolecular interaction networks. Genome Res 13: 2498-2504, 2003.

18. Horvath S and Dong J: Geometric interpretation of gene coexpression network analysis. PLoS Comput Biol 4: e1000117, 2008.

19. Ritchie ME, Phipson B, Wu D, Hu YF, Law CW, Shi W and Smyth GK: Limma powers differential expression analyses for RNA-sequencing and microarray studies. Nucleic Acids Res 43 e47, 2015.

20. Burns C, Bagnall RD, Lam L, Semsarian C and Ingles J: Multiple gene variants in hypertrophic cardiomyopathy in the era of Next-generation sequencing. Circ Cardiovasc Genet 10: e001666, 2017.

21. Han P, Li W, Lin CH, Yang J, Shang C, Nuernberg ST, Jin KK, $\mathrm{Xu} \mathrm{W}$, Lin CY, Lin CJ, et al: A long noncoding RNA protects the heart from pathological hypertrophy. Nature 514: 102-106, 2014.
22. Gomez J, Lorca R, Reguero JR, Martin M, Moris C, Alonso B, Iglesias S, Diaz-Molina B, Avanzas P and Coto E: Genetic variation at the long noncoding RNA H19 gene is associated with the risk of hypertrophic cardiomyopathy. Epigenomics 10: 865-873, 2018.

23. Khatri P, Sirota M and Butte AJ: Ten years of pathway analysis: current approaches and outstanding challenges. PLoS Comput Biol 8: e1002375, 2012.

24. Jin SH, Zhou RH, Guan XY, Zhou JG and Liu JG: Identification of novel key lncRNAs involved in periodontitis by weighted gene co-expression network analysis. J Periodontal Res 55: 96-106, 2020.

25. Gu HY, Yang M, Guo J, Zhang C, Lin LL, Liu Y and Wei RX: Identification of the biomarkers and pathological process of osteoarthritis: Weighted gene co-expression network analysis. Front Physiol 10: 275, 2019.

26. Yin L, Wang Y, Lin Y, Yu G and Xia Q: Explorative analysis of the gene expression profile during liver regeneration of mouse: A microarray-based study. Artif Cells Nanomed Biotechnol 47: 1113-1121, 2019.

This work is licensed under a Creative Commons Attribution-NonCommercial-NoDerivatives 4.0 International (CC BY-NC-ND 4.0) License. 